\title{
Predictive modeling for 14-day unplanned hospital readmission risk by using machine learning algorithms
}

Yu-Tai Lo ${ }^{1 \dagger}$, Jay Chiehen Liao ${ }^{2 \dagger}$, Mei-Hua Chen ${ }^{1}$, Chia-Ming Chang ${ }^{1,3}$ and Cheng-Te Li2 ${ }^{2 *}$

\begin{abstract}
Background: Early unplanned hospital readmissions are associated with increased harm to patients, increased medical costs, and negative hospital reputation. With the identification of at-risk patients, a crucial step toward improving care, appropriate interventions can be adopted to prevent readmission. This study aimed to build machine learning models to predict 14-day unplanned readmissions.

Methods: We conducted a retrospective cohort study on 37,091 consecutive hospitalized adult patients with 55,933 discharges between September 1, 2018, and August 31, 2019, in an 1193-bed university hospital. Patients who were aged $<20$ years, were admitted for cancer-related treatment, participated in clinical trial, were discharged against medical advice, died during admission, or lived abroad were excluded. Predictors for analysis included 7 categories of variables extracted from hospital's medical record dataset. In total, four machine learning algorithms, namely logistic regression, random forest, extreme gradient boosting, and categorical boosting, were used to build classifiers for prediction. The performance of prediction models for 14-day unplanned readmission risk was evaluated using precision, recall, F1-score, area under the receiver operating characteristic curve (AUROC), and area under the precision-recall curve (AUPRC).
\end{abstract}

Results: In total, 24,722 patients were included for the analysis. The mean age of the cohort was $57.34 \pm 18.13$ years. The 14-day unplanned readmission rate was 1.22\%. Among the 4 machine learning algorithms selected, Catboost had the best average performance in fivefold cross-validation (precision: 0.9377 , recall: 0.5333 , F1-score: 0.6780, AUROC: 0.9903, and AUPRC: 0.7515). After incorporating 21 most influential features in the Catboost model, its performance improved (precision: 0.9470, recall: 0.5600, F1-score: 0.7010, AUROC: 0.9909, and AUPRC: 0.7711).

Conclusions: Our models reliably predicted 14-day unplanned readmissions and were explainable. They can be used to identify patients with a high risk of unplanned readmission based on influential features, particularly features related to diagnoses. The operation of the models with physiological indicators also corresponded to clinical experience and literature. Identifying patients at high risk with these models can enable early discharge planning and transitional care to prevent readmissions. Further studies should include additional features that may enable further sensitivity in identifying patients at a risk of early unplanned readmissions.

\footnotetext{
${ }^{*}$ Correspondence: chengte@mail.ncku.edu.tw

${ }^{\dagger}$ Yu-Tai Lo and Jay Chiehen Liao are co-first authors

2 Institute of Data Science, National Cheng Kung University, No. 1,

University Road, Tainan City 701, Taiwan (R.O.C.)

Full list of author information is available at the end of the article
} permitted by statutory regulation or exceeds the permitted use, you will need to obtain permission directly from the copyright holder. To view a copy of this licence, visit http://creativecommons.org/licenses/by/4.0/. The Creative Commons Public Domain Dedication waiver (http://creativecommons.org/publicdomain/zero/1.0/) applies to the data made available in this article, unless otherwise stated in a credit line to the data. 
Keywords: Unplanned readmission, Machine learning, Risk prediction model, Discharge planning, Healthcare quality indicators

\section{Introduction}

Hospital readmissions disrupt the normality of the lives of families and caregivers of patients; moreover, they are associated with harm to patients, reduced quality of care [1], and increases in overall health care costs $[2,3]$. The hospital readmission rate is considered a performance indicator to measure a hospital's quality of care [4]. Furthermore, decreasing unnecessary hospital readmissions can potentially reduce financial and health care burden and improve the quality of care $[5,6]$.

One of the approaches for decreasing the hospital admission rate is to identify patients at risk of readmission; this will enable further investigations, and preventive strategies can then be developed because many readmissions are preventable $[7,8]$. However, diverse and complex factors lead to readmissions, and clinicians cannot process all information to accurately identify at-risk patients [9]. Applying predictive models can direct medical attention toward patients with a high readmission risk, which leverages health care systems and saves health care expenditure.

Current models for readmission risk prediction include attributes describing patient's initial admission; clinical data have been developed and validated for this, but they have yielded moderate discriminative ability [10, 11]. The complex interaction between readmission and potential risk makes accurate prediction of readmission difficult. Machine learning (ML) methods can harness high-dimensional medical data to generate accurate patient risk stratification models and shape health care decisions through the customization of care to individual patients [12].

Preliminary studies have demonstrated that for 30-day all-cause hospital readmission prediction, ML models are better than conventional predictive models [13, 14]. Nevertheless, only unplanned readmissions may lead to substandard care [15]. The likelihood of unplanned readmissions is the highest in the immediate postdischarge period [3], and early 14-day unplanned readmissions were demonstrated to be associated with quality of inpatient care; thus, they were deemed avoidable in cases of high-quality care [1]. Recent studies have shown that readmissions within the first 7 days of hospital discharge may be more preventable than later 30 -day readmissions $[7,16,17]$ and are mostly related to potential gaps in care during the index hospitalization [16, 18]. In Taiwan, the rate of unplanned 14-day readmission for the same or related diagnosis is among the continuous monitoring indicators of care quality of the National Health Insurance Administration; in turn, it affects the hospital accreditation and indirectly influences reimbursement to hospitals [19]. However, whether predictive models and significant predictors of 14-day unplanned hospital readmissions vary from those of 7-day or 30-day unplanned readmissions has not been thoroughly investigated.

Furthermore, predicting readmission early can improve the quality of care. Although ML has been successful with large datasets for predicting 30-day unplanned readmission [12, 20], studies investigating ML-based risk prediction models for identifying high-risk patients for 7- or 14-day unplanned hospital readmission are also lacking.

Therefore, the objective of our study was to build ML models that can accurately predict 14-day unplanned hospital readmissions and to identify influential risk factors in a cohort of patients discharged from a tertiary teaching hospital in Taiwan.

\section{Methods}

\section{Study design and participants}

This retrospective cohort study included consecutive patients discharged from a 1193-bed tertiary care academic medical center in Tainan, Taiwan, from September 1, 2018, to August 31, 2019. Patients who were aged $<20$ years, who were admitted for cancer-related treatment, who participated in pharmaceutical clinical trial, who were discharged against medical advice, who died during admission, or who lived abroad were excluded from the study. The study protocol was approved by the institutional review board of the hospital (A-ER-108-309).

\section{Predictor variables}

Data for analysis included 7 categories of variables extracted from hospital's medical record dataset: (1) demographic characteristics; (2) health care utilization 6 months before index admission; (3) diagnoses 1 year before index admission including the total count of inpatient diagnoses in the past year (we collected 3 major diagnoses of each hospitalization; if the patient was admitted twice 1 year before the index admission, 6 inpatient diagnoses were collected), number of unique inpatient diagnoses in the past year (we deleted duplicate diagnoses from the total inpatient diagnoses), total counts of outpatient diagnoses in the past year, and the number of unique outpatient diagnoses in the past year; (4) overall comorbidity and functional evaluation on 
Table 1 List of variables and their corresponding category utilized in predicting 14-day unplanned readmission risk

\begin{tabular}{|c|c|}
\hline Category & Variable \\
\hline Demographic & Age; Sex; Marital status; Religion; Education; Area of residence; Living alone \\
\hline Health care utilization 6 months before index admission & Number of hospitalizations; Emergency department visits; Outpatient visits \\
\hline Diagnoses 1 year before index admission & $\begin{array}{l}\text { The total count of inpatient diagnoses; Number of unique inpatient diagnoses; } \\
\text { Total counts of outpatient diagnoses; The number of unique outpatient diagnoses }\end{array}$ \\
\hline Overall comorbidity and functional evaluation on index admission & $\begin{array}{l}\text { The } 3 \text { major diagnoses of index admission; Charlson comorbidity index; Depression } \\
\text { diagnoses; Consciousness level; Activities of daily living according to dependency } \\
\text { level in mobility, dressing, feeding, toileting, and bathing; Nutrition status; Mood; } \\
\text { Urinary incontinence; History of fall }\end{array}$ \\
\hline Health care services-related variables during index admission & $\begin{array}{l}\text { Index type of admission; Disease-Related Group of the index admission; Health } \\
\text { education }\end{array}$ \\
\hline One-time laboratory values recorded just before discharge & $\begin{array}{l}\text { Hematocrit; White blood cell count; Red blood cell count; Mean corpuscular } \\
\text { volume; Platelet count; Hemoglobin; Prothrombin time; Blood Urea Nitrogen; Cre- } \\
\text { atinine; Aspartate Aminotransferase; Alanine Aminotransferase; Lactate Dehydroge- } \\
\text { nase; Y-glutamyl transferase; Total Bilirubin; Potassium; Calcium; Sodium; Albumin, } \\
\text { C-reactive protein; Thyroid-Stimulating Hormone }\end{array}$ \\
\hline Discharge-related factors & $\begin{array}{l}\text { Registered in the discharge planning services; Vital signs recorded } 24 \mathrm{~h} \text { before dis- } \\
\text { charge (systolic and diastolic blood pressure, pulse rate, respiratory rate, and body } \\
\text { temperature); Department of discharge; Attending physician's employee identity } \\
\text { and years of experience; Number of discharge medication categories; Total number } \\
\text { of tablets in discharge medication; Discharge destination; Discharge with pressure } \\
\text { injury (or injuries); Types of catheters at discharge; Index hospital length of stay }\end{array}$ \\
\hline
\end{tabular}

index admission including Charlson comorbidity index [21], presence of depression according to International classification of Diseases, Tenth Revision code [22], nutrition status according to Malnutrition Universal Screening Tool [23], and mood status according to Brief Symptom Rating Scale [24]; (5) health care servicesrelated variables during index admission; (6) one-time laboratory values recorded just before discharge; (7) discharge-related variables. The detailed descriptions of all predictor variables are listed in Table 1.

\section{Validation of hospital data}

All variables that constitute the data for analysis were validated through the medical record review of randomly identified patient records at a 1:50 proportion for participants had 14-day unplanned readmission by one of the authors (MHC).

\section{Preprocessing of features}

We grouped certain categories together to reduce the numbers of categories of these features. Assuming that missing values are distributed randomly, we used imputation, which is a common approach for dealing with missing values [25]. Missing values in continuous features were filled with the median values of the features. We did not fill them with the mean values due to the asymmetric distribution of features. As for categorical features, we filled missing values with modes if they had a relatively smaller proportion. Otherwise, we treated missing values as a new category. For binary features, we filled missing values with a negative value.

\section{Study outcome}

The primary outcome was unplanned readmissions within 14 days of discharge after index admission. Unplanned readmission was defined as admission for the same or a related diagnosis according to the National Health Insurance Administration's definition. After thoroughly evaluating readmitted patients' diagnoses for readmission and their clinical courses, the attending physicians in charge of the readmissions made the distinction of unplanned readmissions.

\section{ML techniques}

We used $4 \mathrm{ML}$ algorithms, namely logistic regression [26], random forest (RF) [27], extreme gradient boosting (Xgboost) [28], and gradient boosting with categorical features support (Catboost) [29], to build classifiers for prediction. Logistic regression is a traditional statistical model and usually used to be the baseline to compare to ML models [13]. RF, Xgboost, and Catboost have shown acceptable performance in predicting unplanned readmissions in previous studies [30].

To address the assumption of non-collinearity for logistic regression, we computed Variance Inflation Factor (VIF) values to detect if collinearity existed and removed features with collinearity. First, we computed VIF values for each feature. Then we removed the feature with 
the highest VIF value and compute VIF values again and repeated this procedure until all VIF values are smaller than 4.

Feature importance can be obtained with RF, Xgboost, and Catboost models based on their use of features during training. Feature importance demonstrates how much the prediction changes as the feature values vary. Higher feature importance indicates the higher importance of the feature to the model prediction. Through feature selection based on feature importance, we selected the most influential features to enhance the model's generalizability and performance and make the model practically usable. We set several thresholds of feature importance and chose values that provided an appropriate number of features the model.

Models were trained using Python 3.6.9 on a Linux Intel Xenon Gold 6138 processor with $2.0 \mathrm{GHz}$ RAM and a 450G CPU. An NVIDIA Tesla V100 32 GB GPU was used to speed up the training process for Catboost models. We implemented models with Scikit-Learn, Xgboost, and Catboost packages of Python. Model hyperparameters not learnable during training and determined the structure of models, were set as the defaulted values of these packages.

\section{Training and evaluation}

The dataset was split into training (75\%) and testing (25\%) sets with stratified random sampling to fix the proportion of patients with unplanned readmission in both subsets. The predictive models for readmission were trained on the training set and were applied and evaluated on the testing set. The fivefold cross-validation approach was used to obtain reliable results for evaluating prediction models or for obtaining reliable results. The original training set was split into 5 folds through stratified random sampling. For the $i$ th iteration, fold $i$ was treated as the validation set and the remaining 4 folds were used to train the model. The model was evaluated using the validation set. The procedure was repeated for 5 iterations. Evaluation results of 5 iterations were collected to compute the mean value and standard deviation.

We used 5 commonly used evaluation indices to evaluate the models, namely precision score (positive predict rate), recall score (sensitivity), F1-score, area under the receiver operating characteristic curve (AUROC), and area under the precision-recall curve (AUPRC). These evaluation indices are commonly reported in the evaluation of classification problems with ML [31]. Furthermore, AUPRC is appropriate for prediction tasks with a low rate of positive cases [32]. Their definitions are listed in Table 2.

\section{Model interpretation}

We adapted SHapley Additive exPlanations (SHAP) [33], a game theory-based framework with feature importance calculation, to interpret our ML model. It assigns an importance value (SHAP value) to each feature to explain the predication of each observation. It can also summarize how every feature contribute to the prediction. To calculate SHAP values on categorical features, the approach to turn every category in a categorical feature into dummy variables, namely "one-hot encoding", is utilized naturally.

\section{Results}

\section{Cohort characteristics}

From September 1, 2018, to August 31, 2019, a total of 37, 091 adult patients were discharged, with a total of 55, 933

Table 2 Definitions of evaluation metrics

\begin{tabular}{|c|c|}
\hline $\begin{array}{l}\text { Notation/ } \\
\text { evaluation } \\
\text { index }\end{array}$ & Description/definition \\
\hline$T P$ & $\begin{array}{l}\text { True positive. The number of patients who had unplanned readmission and were also predicted to have unplanned readmission by the } \\
\text { model }\end{array}$ \\
\hline FP & $\begin{array}{l}\text { False positive. The number of patients who did not have unplanned readmission but were predicted to have unplanned readmission by } \\
\text { the model }\end{array}$ \\
\hline$T N$ & $\begin{array}{l}\text { True negative. The number of patients who did not have unplanned readmission and were also not predicted to have unplanned read- } \\
\text { mission by the model }\end{array}$ \\
\hline FN & $\begin{array}{l}\text { False negative. The number of patients who had unplanned readmission but were not predicted to have unplanned readmission by the } \\
\text { model }\end{array}$ \\
\hline Precision & $T P /(T P+F P)$ \\
\hline Recall & $T P /(T P+F N)$ \\
\hline F1-score & The harmonic mean of precision and recall. The formula is as follows: $F 1=2 /(1 /$ Precision $+1 /$ Recall $)$ \\
\hline AUROC & Area under the receiver operating characteristic curve \\
\hline AUPRC & Area under the precision-recall curve \\
\hline
\end{tabular}


From Sep 1, 2018, through Aug 31, 2019,

a total of 55933 discharges

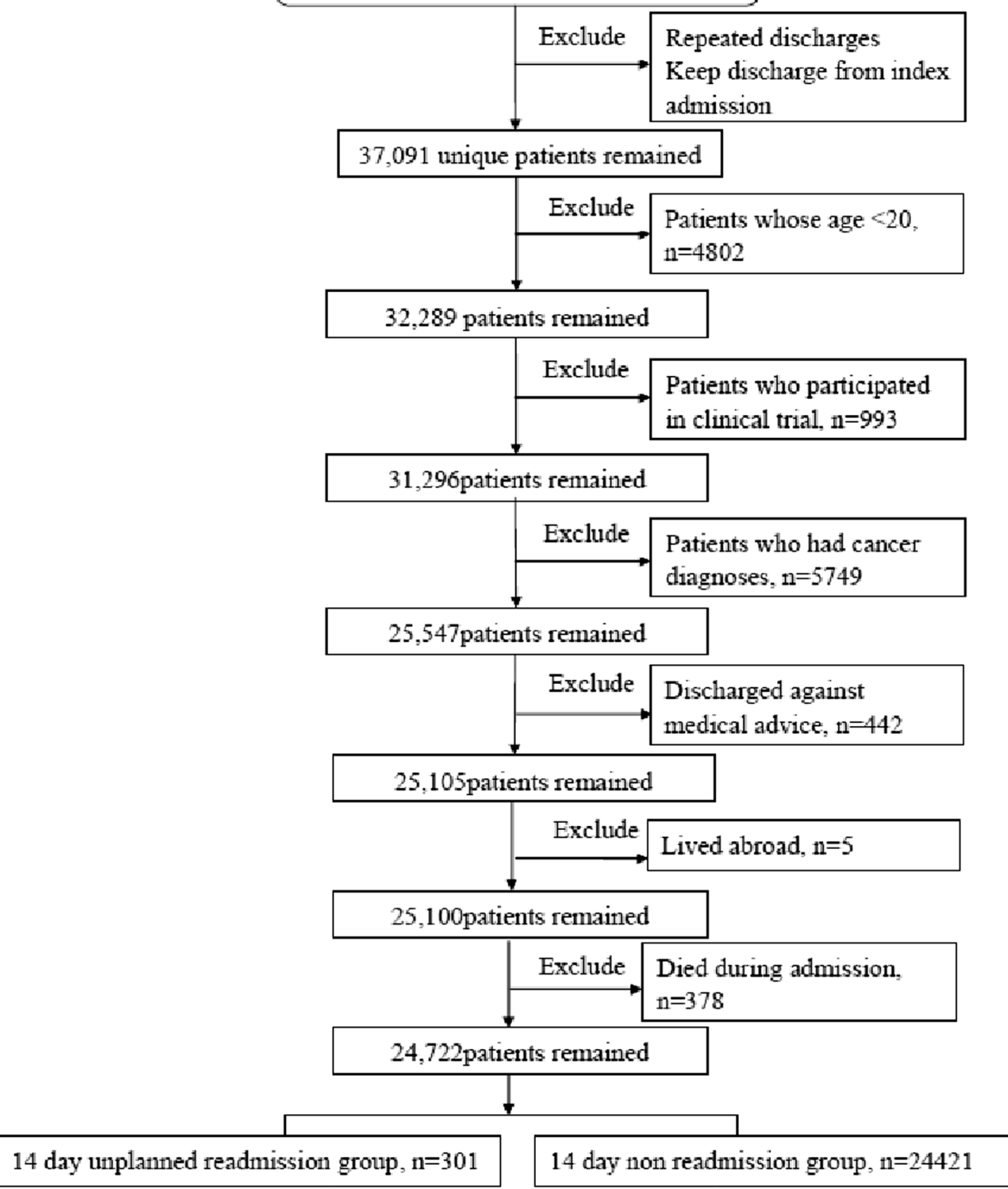

Fig. 1 Flowchart of study cohort selection

discharges (including repeated admissions). Of these, 530 patients had unplanned readmission and 31, 759 patients had no unplanned readmission (unplanned readmission rate: 1.64\%). After exclusion, 301 and 24, 421 patients with and without unplanned readmission were included (unplanned readmission rate: $1.22 \%$ ). The mean age of the cohort was $57.34 \pm 18.13$ years. The training and validation cohorts consisted of 24, 722 patients (Fig. 1).
Characteristics, including laboratory values, between patients with and without unplanned readmission are presented in Additional file 1.

\section{ML model performance}

By computing VIF values, we found that collinearity existed in the 70 original features. We remained 27 features without collinearity $(\mathrm{VIF}<4)$ and created a logistic regression model with these predictors (Additional 
Table 3 Performance metrics of the LACE model and machine learning models based on the testing set with fivefold cross-validation (Mean \pm Standard Deviation, Unit: \%)

\begin{tabular}{lccccc}
\hline Model (\#Features) & Precision & Recall & F1-Score & AUROC & AUPRC \\
\hline LACE (4) & $2.97 \pm 0.15$ & $68.67 \pm 3.86$ & $5.70 \pm 0.29$ & $70.58 \pm 1.88$ & $34.63 \pm 0.00$ \\
Logistic Regression: original features (70) & $45.76 \pm 15.72$ & $4.00 \pm 2.00$ & $7.35 \pm 3.59$ & $80.46 \pm 2.43$ & $10.26 \pm 2.23$ \\
Logistic Regression: original features (27) & $43.62 \pm 20.73$ & $5.00 \pm 1.05$ & $8.84 \pm 2.00$ & $82.88 \pm 3.57$ & $11.66 \pm 3.54$ \\
Random Forest: original features (70) & $100.00 \pm 0.00$ & $41.33 \pm 3.86$ & $58.39 \pm 3.79$ & $97.89 \pm 0.71$ & $70.15 \pm 4.23$ \\
Xgboost: original features (70) & $93.23 \pm 5.35$ & $45.67 \pm 3.89$ & $61.25 \pm 4.32$ & $97.95 \pm 0.52$ & $66.52 \pm 2.23$ \\
Catboost 1 (C1): original features (70) & $93.77 \pm 4.05$ & $53.33 \pm 5.27$ & $67.80 \pm 4.47$ & $99.03 \pm 0.07$ & $75.15 \pm 1.92$ \\
Catboost 2: features in C1 with importance > 0.5 (35) & $95.12 \pm 2.54$ & $56.00 \pm 5.33$ & $70.29 \pm 3.84$ & $99.04 \pm 0.09$ & $76.11 \pm 2.45$ \\
Catboost 3: features in C1 with importance > 0.6 (28) & $95.09 \pm 3.09$ & $55.33 \pm 5.31$ & $69.74 \pm 3.99$ & $99.08 \pm 0.08$ & $76.69 \pm 1.85$ \\
Catboost 4: features in C1 with importance > 0.8 (21) & $94.70 \pm 3.52$ & $56.00 \pm 6.02$ & $70.10 \pm 4.40$ & $99.09 \pm 0.08$ & $77.11 \pm 1.93$ \\
Catboost 5: features in C1 with importance > 0.9 (19) & $93.20 \pm 1.59$ & $55.33 \pm 5.72$ & $69.29 \pm 4.76$ & $99.07 \pm 0.10$ & $76.80 \pm 1.64$ \\
Catboost 6: features in C1 with importance > 1.1 (14) & $91.46 \pm 2.12$ & $56.67 \pm 4.47$ & $69.86 \pm 3.51$ & $99.00 \pm 0.11$ & $76.97 \pm 2.90$ \\
\hline
\end{tabular}

$\overline{A U R O C}=$ area under the receiver operating characteristic curve; $A U P R C=$ area under the precision-recall curve

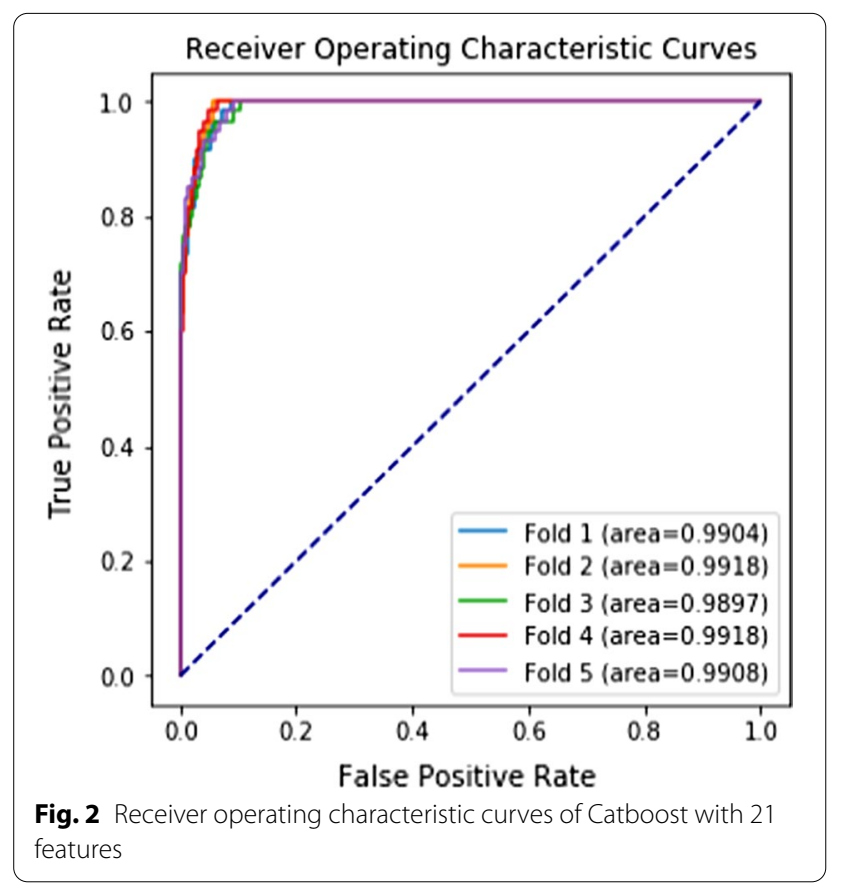

file 2). Among the $4 \mathrm{ML}$ algorithms, the logistic regression model had the worst performance, and Catboost had the best performance. Table 3 lists the performance results of models evaluated on the testing set and the different performance results of Catboost models during the feature selection process. After feature selection, the Catboost model with 21 features (Catboost 4 in Table 3) performed the best in terms of AUROC (0.9909) and AUPRC (0.7711), which considered both positive rate and sensitivity. If more features were removed (Catboost 5 and 6 in Table 3), the precision

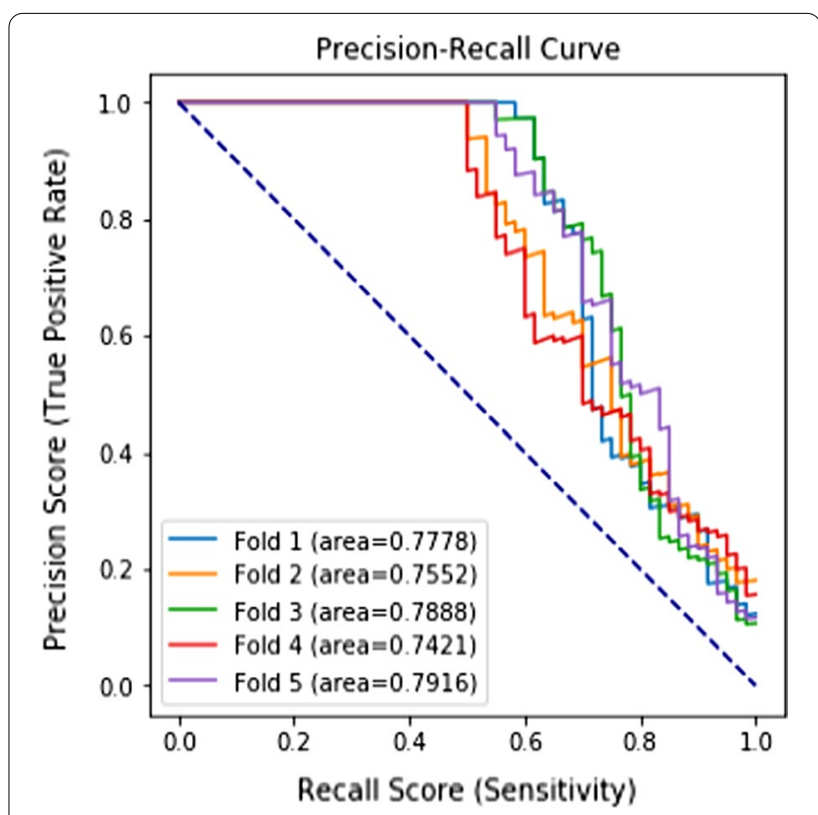

Fig. 3 Precision-Recall Curves of Catboost with 21 features

score decreased. Therefore, we adopted Catboost 4 with 21 features as the final model. Figures 2 and 3 present its receiver operating characteristic curve and precision-recall curve.

\section{Significant predictors}

The final Catboost model has 21 useful features. Figure 4 shows the importance of these features with the average value and standard deviation in 5 -fold cross-validation. 


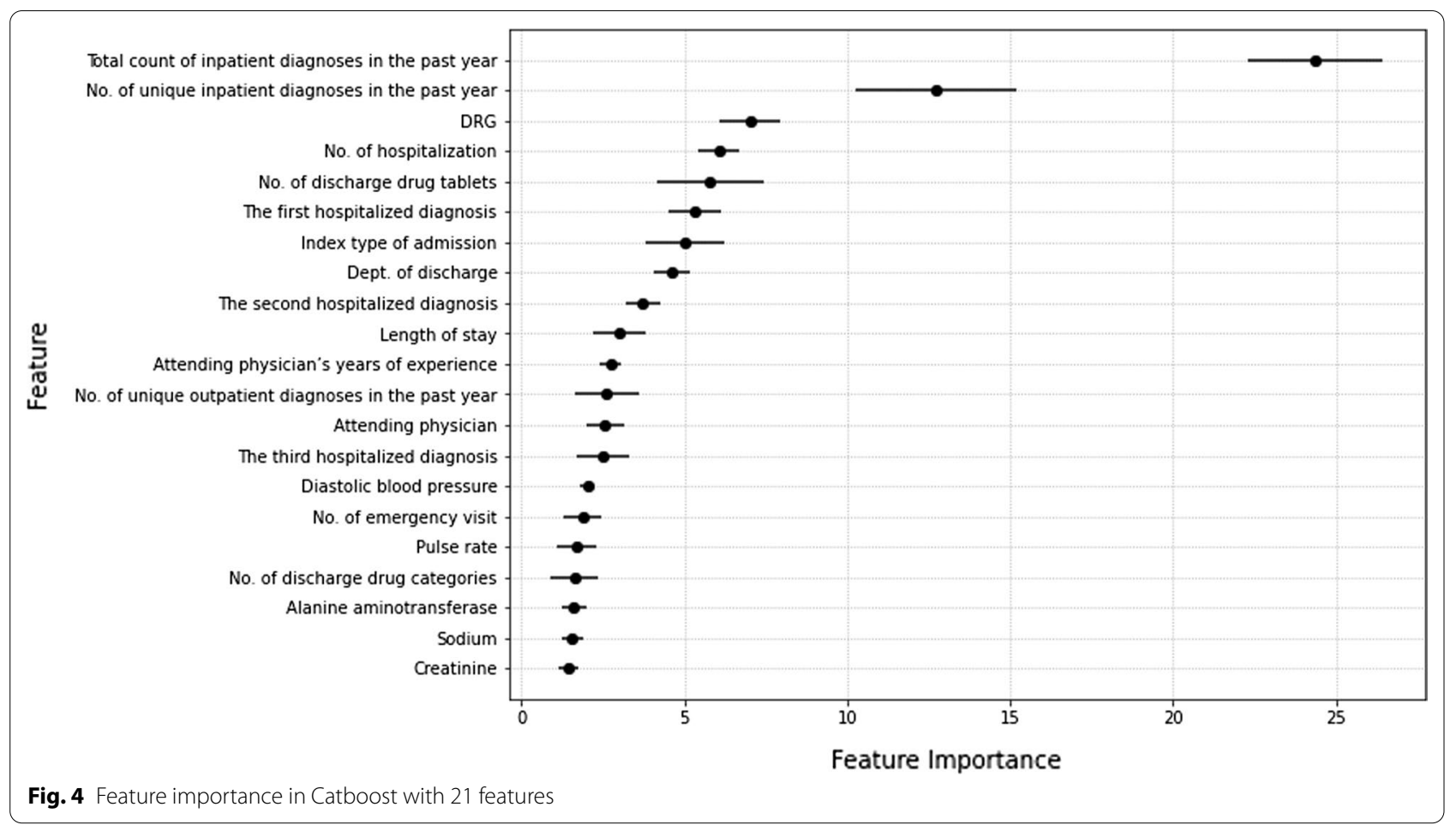

Total count of inpatient diagnoses in the past year No. of unique inpatient diagnoses in the past year

DRG

No. of hospitalization No. of discharge drug tablets The first major hospitalized diagnosis Index type of admission Department of discharge The second major hospitalized diagnosis Length of stay Attending physician's years of experience No. of unique outpatient diagnoses in the past year Attending physician

The third major hospitalized diagnosis Diastolic blood pressure No. of emergency visit Pulse rate No. of discharge drug categories Alanine aminotransferase Sodium Creatinine

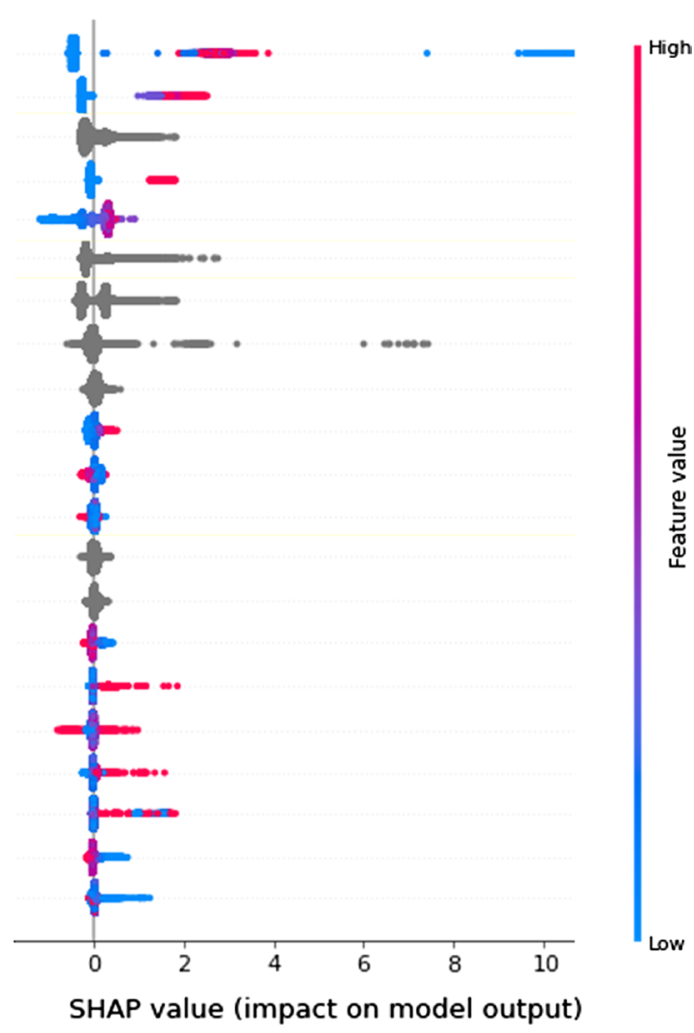

Fig. 5 Association between feature value and SHAP value in Catboost with 21 features 

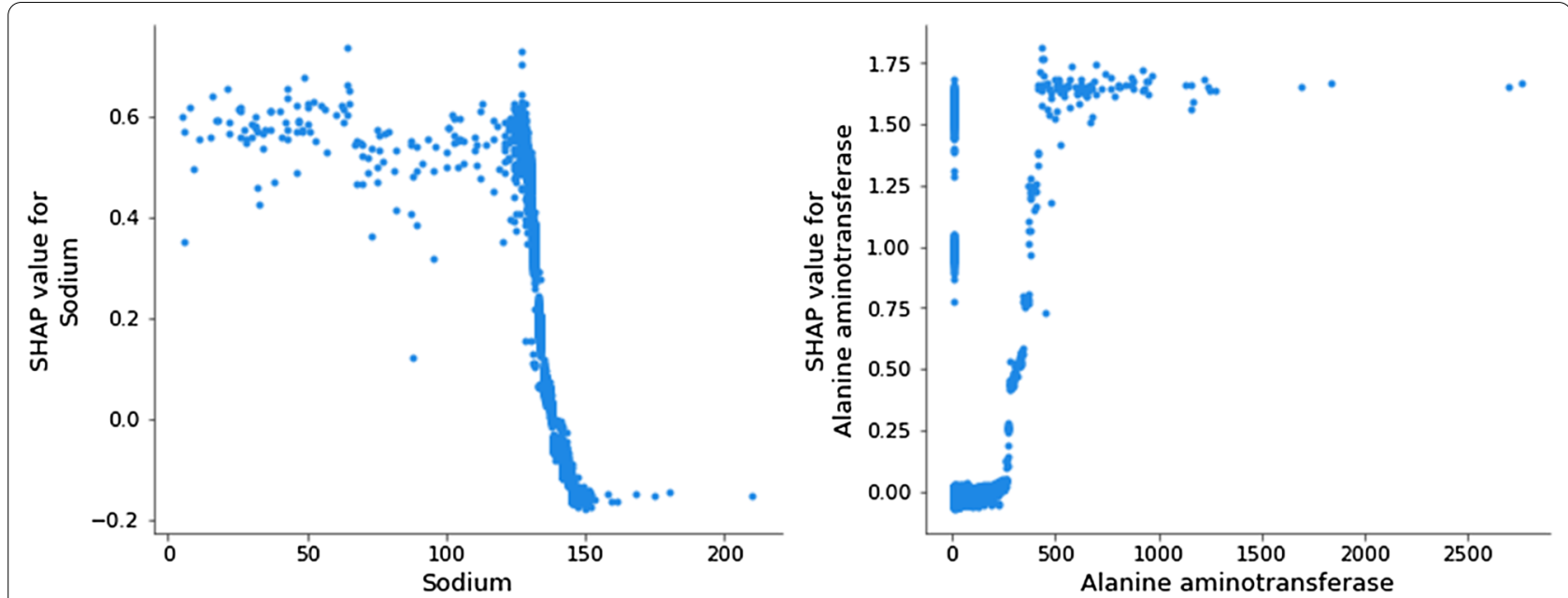

Fig. 6 Association of SHAP value with Sodium (Left) and Alanine aminotransferase (Right) in Catboost with 21 features

\section{Model interpretation}

In Figs. 5 and 6, SHAP values are used to demonstrate how our Catboost model operates to classify patients as cases with 21 features. Among training sets in 5 folds, we randomly take one to demonstrate.

In Fig. 5, red points refer relatively higher values and blue points refer relatively lower values in numerical features. For categorical features, one grey point represents a category in the given feature. And its location on the horizontal axis indicates the SHAP value that patients who belong to this category would be assigned from this feature. Overall, the model yielded a higher probability of unplanned readmission to patients with more inpatient diagnoses or higher numbers of unique inpatient diagnoses 1 year before index admission, indicating that these patients are more likely to have unplanned readmissions. In addition, the distribution of grey points of feature "DRG" is wider than that of feature "Attending physician", meaning that the variation of SHAP values of different DRGs is higher than that of different attending physicians. That is, compared to different attending physicians, different DRGs may contribute larger difference of unplanned readmission possibility. This corresponds to Fig. 4, which shows that the average feature importance of "DRG" is higher than that of "Attending physician" and that their bars of one standard deviation even do not overlap ( $7.02 \pm 0.93$ vs. $2.57 \pm 0.58$ ). Figure 6 shows the association of SHAP values with values of 2 physiological features, namely sodium and alanine aminotransferase.

\section{Discussion}

Although recent studies have used ML to predict 30-day all-cause or unplanned readmission risk, analyses for predicting 14-day unplanned readmission remain rare in the literature. To the best of our knowledge, this is the first study to use ML to predict 14-day unplanned readmission and to select features to establish prediction models and the first study to use ML for predicting unplanned readmissions by using local data in Taiwan.

Accurately identifying patients at risk of unplanned readmission shortly after discharge can enable early discharge planning and transitional care to prevent recurrent readmissions. Our findings demonstrated that ML algorithms can predict a patient's risk of 14-day unplanned readmission with good discrimination and precision. They also suggested that unlike conventional approaches such as logistic regression, other ML algorithms have the advantage of convenient utility besides accurate prediction. That is, we do not need to consider collinearity of predictors when fitting an ML model such as Catboost.

The most important finding is that the final ML model demonstrated good discrimination (AUROC $>0.99$ and AUPRC > 0.77) with excellent precision (0.9470) and moderate sensitivity (0.5600). Furthermore, we computed the LACE score (calculated at discharge using 4 items: length of stay, acuity of admission, comorbidities and emergent department visits 6 months before index admission) [34], a well-known readmission risk assessment score [34], in our cohort. Although LACE had a relatively high sensitivity (0.6867), its precision score was extremely low. Our final ML model strongly outperformed LACE in terms of evaluation metrics except for the recall score (precision: 0.9470 vs 0.0297, F1-score: 0.7010 vs 0.0570 , AUROC: 0.9909 vs 0.7058 , and AUPRC: 0.7711 vs 0.3463$)$. Besides LACE, according to previous reports, our ML model seemed to outperform other 
well-known developed readmission risk assessment scores, such as PARR-30 [35] (calculated with age, place of residence, acuity of admission, emergent department visits in the last year, history in the prior two years of 11 major health conditions drawn from the Charlson co-morbidity index, and the hospital of admission), and HOSPITAL score [36] (calculated with hemoglobin, discharge from an oncology service, sodium level, procedure during the index admission, index type of admission, number of admissions during the last year, and length of stay). The performance of PARR-30 and HOSPITAL were AUROC: PARR-30 $=0.7$, HOSPITAL: 0.72; precision: PARR- $30=0.59$; sensitivity: PARR- $30=0.054)$. The benefit of ML is that it is trained for each hospital and weighted for individual characteristics. Furthermore, several studies have demonstrated that ML models are better than conventional models for all-cause hospital readmission prediction [13, 14, 30, 37, 38], and some studies have evaluated models for unplanned admissions [12, 20,39]. Goyal et al. used a national database of 59, 145 patients who underwent spinal fusion to evaluate seven ML algorithms, and all models showed moderate performance with 30-day unplanned readmission (AUROC: 0.630.66, sensitivity: $0.46-0.64$, and precision: 0.07 ). Among the seven ML models, gradient boosting machines performed the best [12]. Morgan et al. compared ML with conventional risk prediction scores for 30-day unplanned readmissions in 14,062 patients at 3 different hospitals, and ML score predicted readmissions better than conventional scores (AUROC: 0.81, precision: 0.375, and sensitivity: 0.283) [20]. Considering overall performance, our final model seems to have satisfying prediction precision and sensitivity. A likely explanation for the satisfying performance of ML in our study is that we adopted Catboost, a ML algorithm designed for processing categorical data. Among 21 features in the final model, 6 of them are categorical features. Furthermore, the numbers of categories of features were extremely large in our data (e.g., 684 in DRG and 297 in attending physicians' employee identity), which may be difficult for non-Catboost models to deal with.

The second important finding is that our ML prediction model successfully identified several useful predictors, which have also been used in the conventional risk assessment scores, such as LACE, PARR-30, and HOSPITAL. In the present model, patient age and place of residence were not associated with readmission risk, but hospitalizations 6 months before index admission was an important feature. This finding is consistent with the results of an updated systemic review regarding prediction models of 28- or 30-day unplanned hospital readmissions, which showed that the number of previous admissions ranks the fourth among top 10 most important variables and is included in 29 unplanned readmission prediction models [10]. Furthermore, the number of total discharge medication tablets and medication categories were influential features in our model. Prescribed drug-related readmissions represent a nonnegligible proportion of readmissions, particularly among older patients [30, 40, 41]. Our study did not include high-risk medications as a predictor variable, and future investigation is warranted to understand the effect of different medications on 14-day readmission risk.

The strength of this study is that it includes multiple predictor variables from demographic characteristics, prior health care utilization, diagnosis-related variables, overall health and function assessment on the index admission, variables related to healthcare services during admission, laboratory tests on discharge, and dischargerelated variables for analysis. Among the 21 features in the final model, most of the predictors could be identified at an early stage of admission; therefore, these variables can be used to estimate the probability of readmission soon after patients are admitted [37]. Other variables may not be modified by actions taken at discharge, but the most effective interventions preventing readmission in fact are related to postdischarge support to patients and caregivers [42]. By using the prediction model, patients identified as at-risk can be closely monitored and early outpatient follow-up or referrals to home health care services can be arranged.

This study has several limitations. First, data were retrospectively extracted from medical records, which may have reduced our ability to identify all risk factors for readmission. Second, we did not consider readmission to another facility because the data were limited to readmissions in the same hospital. Third, this study involved patients of a single academic tertiary hospital, and our findings may not be generalizable to other facilities; hence, further external validation is required. Finally, we used cross-sectional features; we lacked features with sequential or temporal trajectory of events in electronic health records over time, which contains important information about disease progression and patient status. Access to large volumes of patient records with a sequential trajectory of events, such as electronic health records, warrants further investigation to improve prediction sensitivity and performance. Nevertheless, our findings could lay the groundwork for future studies using ML as a risk stratification tool for early unplanned readmissions. 


\section{Conclusions}

ML prediction models can help clinicians to accurately identify patients likely to experience early unplanned readmission. Our study results enable clinicians to identify patients at a high risk of hospital readmission and also suggest interventions that can be initiated during hospitalization, such as providing adequate patient or family education before discharge. Although our work has scope for improvement, we believe that it has set the stage for further research to improve the accuracy of predicting early readmission risk.

\begin{abstract}
Abbreviations
ML: Machine learning; DRG: Disease-related group; RF: Random forest; Xgboost: Extreme gradient boosting; Catboost: Gradient boosting with categorical features; AUROC: Area under the receiver operating characteristic curve; AUPRC: Area under the precision-recall curve; SHAP: SHapley additive exPlanations.
\end{abstract}

\section{Supplementary Information}

The online version contains supplementary material available at https://doi. org/10.1186/s12911-021-01639-y.

Additional file 1. Numbers and proportions of missing values in study variables.

Additional file 2. The Variance Inflation Factor Values of 27 Features Included in the Final Logistic Regression Model.

\section{Acknowledgements}

We thank the nursing supervisor of discharge planning Ms. Hsiu-Hua Lee, discharge planning nurses, and the information technicians at National Cheng Kung University Hospital for helping us collect data from patients' medical records. This work is supported by the Ministry of Science and Technology (MOST) of Taiwan under grants 109-2636-E-006-017 (MOST Young Scholar Fellowship), 110-2221-E-006-001, 110-2221-E-006-136-MY3, and 110-2634-F-002-051.

\section{Authors' contributions}

YTL, JCL and CTL conceptualized the study. YTL and JCL were the major contributors in writing the first draft of the manuscript. YTL and MHC collected data and JCL performed the analysis. CMC and CTL provided constructive feedback of the draft protocol and manuscript documents. All authors read and approved the final manuscript.

\section{Funding}

This study was supported by the germination program of the National Cheng Kung University and in part by grants from National Cheng Kung University Hospital (NCKUH-10909041; NCKUH-1 1009005; NCKUH-1 1003007), Taiwan. The Funders had no role in study design, data collection and analysis, decision to publish, or preparation of the manuscript.

\section{Declarations}

Ethics approval and consent to participate

This study was ethically approved by the institutional review board of the National Cheng Kung University Hospital (A-ER-109-469). In view of the retrospective nature, the need for individual patient consent was waived by the Institutional Review Board of National Cheng Kung University Hospital as data protection safeguard was in place. This study was performed in accordance with the Declaration of Helsinki.

\section{Consent for publication}

Not applicable.

\section{Availability of data and materials}

Data and resources will be shared with other eligible investigators through academically established means. The datasets used and analyzed during the study will be available from the corresponding author on reasonable request.

\section{Competing interests}

The authors declare that they have no competing interests.

\section{Author details}

${ }^{1}$ Department of Geriatrics and Gerontology, National Cheng Kung University Hospital, College of Medicine, National Cheng Kung University, Tainan, Taiwan (R.O.C.). ${ }^{2}$ Institute of Data Science, National Cheng Kung University, No. 1, University Road, Tainan City 701, Taiwan (R.O.C.). ${ }^{3}$ Department of Medicine and Institute of Gerontology, College of Medicine, National Cheng Kung University, Tainan, Taiwan (R.O.C.)

Received: 22 June 2021 Accepted: 22 September 2021

Published: 20 October 2021

\section{References}

1. Ashton CM, Kuykendall DH, Johnson ML, Wray NP, Wu L. The association between the quality of inpatient care and early readmission. Ann Intern Med. 1995;122(6):415-21.

2. Joynt KE, Jha AK. Thirty-day readmissions-truth and consequences. N Engl J Med. 2012;366(15):1366-9.

3. Jencks SF, Williams MV, Coleman EA. Rehospitalizations among patients in the Medicare fee-for-service program. N Engl J Med. 2009:360(14):1418-28.

4. Stefan MS, Pekow PS, Nsa W, Priya A, Miller LE, Bratzler DW, et al. Hospital performance measures and 30-day readmission rates. J Gen Intern Med. 2013;28(3):377-85.

5. Berenson RA, Paulus RA, Kalman NS. Medicare's readmissions-reduction program - a positive alternative. N Engl J Med. 2012;366(15):1364-6.

6. Epstein AM, Jha AK, Orav EJ. The relationship between hospital admission rates and rehospitalizations. N Engl J Med. 2011;365(24):2287-95.

7. Auerbach AD, Kripalani S, Vasilevskis EE, Neil S, Lindenauer PK, Metlay JP, et al. Preventability and causes of readmissions in a national cohort of general medicine patients. JAMA Intern Med. 2016;176(4):484-93.

8. Vest JR, Gamm LD, Oxford BA, Gonzalez MI, Slawson KM. Determinants of preventable readmissions in the United States: a systematic review. Implement Sci. 2010:5:88.

9. Allaudeen N, Schnipper JL, Orav EJ, Wachter RM, Vidyarthi AR. Inability of providers to predict unplanned readmissions. J Gen Intern Med. 2011;26(7):771-6.

10. Zhou H, Della PR, Roberts P, Goh L, Dhaliwal SS. Utility of models to predict 28-day or 30-day unplanned hospital readmissions: an updated systematic review. BMJ Open. 2016;6(6):e011060.

11. Kansagara D, Englander $H$, Salanitro A, Kagen D, Theobald C, Freeman M, et al. Risk prediction models for hospital readmission: a systematic review. JAMA. 2011;306(15):1688-98.

12. Goyal A, Ngufor C, Kerezoudis P, McCutcheon B, Storlie C, Bydon M. Can machine learning algorithms accurately predict discharge to nonhome facility and early unplanned readmissions following spinal fusion? Analysis of a national surgical registry. J Neurosurg Spine. 2019:1-11.

13. Frizzell JD, Liang L, Schulte PJ, Yancy CW, Heidenreich PA, Hernandez AF, et al. Prediction of 30-day all-cause readmissions in patients hospitalized for heart failure: comparison of machine learning and other statistical approaches. JAMA Cardiol. 2017;2(2):204-9.

14. Zhang Z, Qiu H, Li W, Chen Y. A stacking-based model for predicting 30-day all-cause hospital readmissions of patients with acute myocardial infarction. BMC Med Inform Decis Mak. 2020;20(1):335.

15. Kossovsky MP, Sarasin FP, Bolla F, Gaspoz JM, Borst F. Distinction between planned and unplanned readmissions following discharge from a Department of Internal Medicine. Methods Inf Med. 1999;38(2):140-3.

16. Graham KL, Auerbach AD, Schnipper JL, Flanders SA, Kim CS, Robinson EJ, et al. Preventability of early versus late hospital readmissions 
in a national cohort of general medicine patients. Ann Intern Med. 2018;168(11):766-74.

17. Graham KL, Wilker EH, Howell MD, Davis RB, Marcantonio ER. Differences between early and late readmissions among patients: a cohort study. Ann Intern Med. 2015;162(11):741-9.

18. Saleh SN, Makam AN, Halm EA, Nguyen OK. Can we predict early 7-day readmissions using a standard 30-day hospital readmission risk prediction model? BMC Med Inform Decis Mak. 2020;20(1):227.

19. Chiu WT, Yang CM, Lin HW, Chu TB. Development and implementation of a nationwide health care quality indicator system in Taiwan. Intern J Qual Health Care. 2007;19(1):21-8.

20. Morgan DJ, Bame B, Zimand P, Dooley P, Thom KA, Harris AD, et al. Assessment of machine learning vs standard prediction rules for predicting hospital readmissions. JAMA Netw Open. 2019;2(3):e190348.

21. Charlson ME, Pompei P, Ales KL, MacKenzie CR. A new method of classifying prognostic comorbidity in longitudinal studies: development and validation. J Chronic Dis. 1987;40(5):373-83.

22. Steindel SJ. International classification of diseases, 10th edition, clinical modification and procedure coding system: descriptive overview of the next generation HIPAA code sets. J Am Med Inform Assoc. 2010;17(3):274-82.

23. Stratton RJ, Hackston A, Longmore D, Dixon R, Price S, Stroud M, et al. Malnutrition in hospital outpatients and inpatients: prevalence, concurrent validity and ease of use of the "malnutrition universal screening tool" ('MUST') for adults. Br J Nutr. 2004;92(5):799-808.

24. Chen HC, Wu CH, Lee YJ, Liao SC, Lee MB. Validity of the five-item Brief Symptom Rating Scale among subjects admitted for general health screening. J Formos Med Assoc. 2005;104(11):824-9.

25. Hastie T, Tibshirani R, Friedman J. The Elements of statistical learning: data mining, inference, and prediction. 2nd ed. New York: Springer; 2009.

26. Wright RE. Logistic regression. In: Reading and understanding multivariate statistics. Washington, DC, US: American Psychological Association; 1995: 217-244

27. Breiman L. Random forests. Mach Learn. 2001;45(1):5-32.

28. Chen T, Guestrin C. Xgboost: a scalable tree boosting system. In: the 22nd ACM SIGKDD international conference on knowledge discovery and data mining. 2016

29. Prokhorenkova L, Gusev G, Vorobev A, Dorogush AV, Gulin A. CatBoost: unbiased boosting with categorical features. In: 32nd conference on neural information processing systems (NeurIPS); NeurIPS; 2018.

30. Mohanty SD, McCoy TP, Manda P, Lekan D, Jenkins M. A multi-modal machine learning approach towards predicting patient readmission. In: 2020 International conference on bioinformatics and biomedicine (BIBM). IEEE; 2020.

31. Hossin M, Sulaiman MN. A review on evaluation metrics for data classification evaluations. IJDKP. 2015;5(2):1-11.

32. Ozenne B, Subtil F, Maucort-Boulch D. The precision-recall curve overcame the optimism of the receiver operating characteristic curve in rare diseases. J Clin Epidemiol. 2015:68(8):855-9.

33. Lundberg S, Lee S-I. A unified approach to interpreting model predictions. In: 31st Conference on neural information processing systems (NIPS). 2017.

34. van Walraven C, Dhalla IA, Bell C, Etchells E, Stiell IG, Zarnke K, et al. Derivation and validation of an index to predict early death or unplanned readmission after discharge from hospital to the community. CMAJ. 2010;182(6):551-7.

35. Billings J, Blunt I, Steventon A, Georghiou T, Lewis G, Bardsley M. Development of a predictive model to identify inpatients at risk of re-admission within 30 days of discharge (PARR-30). BMJ Open. 2012;2(4):e001667.

36. Donzé JD, Williams MV, Robinson EJ, Zimlichman E, Aujesky D, Vasilevskis EE, et al. International validity of the hospital score to predict 30-day potentially avoidable hospital readmissions. JAMA Intern Med. 2016;176(4):496-502.

37. Hung LC, Sung SF, Hu YH. A machine learning approach to predicting readmission or mortality in patients hospitalized for stroke or transient ischemic attack. Appl Sci. 2020;10(18):6337.

38. Futoma J, Morris J, Lucas J. A comparison of models for predicting early hospital readmissions. J Biomed Inform. 2015;56:229-38.

39. Beecy AN, Gummalla M, Sholle E, Xu Z, Zhang Y, Michalak K, et al. Utilizing electronic health data and machine learning for the prediction of 30-day unplanned readmission or all-cause mortality in heart failure. Cardiovasc Digit Health J. 2020;1(2):71-9.

40. Morandi A, Bellelli G, Vasilevskis EE, Turco R, Guerini F, Torpilliesi T, et al. Predictors of rehospitalization among elderly patients admitted to a rehabilitation hospital: the role of polypharmacy, functional status, and length of stay. J Am Med Dir Assoc. 2013;14(10):761-7.

41. Bonnet-Zamponi D, d'Arailh L, Konrat C, Delpierre S, Lieberherr D, Lemaire $A$, et al. Drug-related readmissions to medical units of older adults discharged from acute geriatric units: results of the Optimization of Medication in AGEd multicenter randomized controlled trial. J Am Geriatr Soc. 2013;61(1):113-21.

42. Leppin AL, Gionfriddo MR, Kessler M, Brito JP, Mair FS, Gallacher K, et al. Preventing 30-day hospital readmissions: a systematic review and metaanalysis of randomized trials. JAMA Intern Med. 2014;174(7):1095-107.

\section{Publisher's Note}

Springer Nature remains neutral with regard to jurisdictional claims in published maps and institutional affiliations. 